INPLASY

PROTOCOL

To cite: Zeng et al. Prevalence and stability of subthreshold anxiety disorder in adult: a meta analysis. Inplasy protocol 202070025. doi:

10.37766/inplasy2020.7.0025

Received: 08 July 2020

Published: 08 July 2020

Corresponding author:

Cong Zeng

zengcong1995@163.com

Author Affiliation:

Harbin Medical University

Support: NO

Review Stage at time of this submission: The review has not yet started.

Conflicts of interest: NO.

\section{Prevalence and stability of subthreshold anxiety disorder in adult: a meta analysis}

\author{
Zeng, C1; Yang, X2; Cao, J3.
}

Review question / Objective: Subthreshold anxiety disorder refers to people who do not meet all the diagnosis standard of anxiety disorders but are accompanied by functional impairment. However, no study has reported the prevalence of subthreshold anxiety disorder and the stability of prevalence of subthreshold anxiety disorder.

Condition being studied: Anxiety disorder including specific phobia or social anxiety disorder or agoraphobia or panic disorder or panic disorders or generalized anxiety disorder or pediatric anxiety or pediatric anxiety disorder or separation anxiety disorder

INPLASY registration number: This protocol was registered with the International Platform of Registered Systematic Review and Meta-Analysis Protocols (INPLASY) on 08 July 2020 and was last updated on 08 July 2020 (registration number INPLASY202070025).

\section{INTRODUCTION}

Review question / Objective: Subthreshold anxiety disorder refers to people who do not meet all the diagnosis standard of anxiety disorders but are accompanied by functional impairment.However, no study has reported the prevalence of subthreshold anxiety disorder and the stability of prevalence of subthreshold anxiety disorder.

Condition being studied: Anxiety disorder including specific phobia or social anxiety disorder or agoraphobia or panic disorder or panic disorders or generalized anxiety disorder or pediatric anxiety or pediatric 
anxiety disorder or separation anxiety disorder

\section{METHODS}

Participant or population: Types of study to be included: 1.The language of the article is English; 2.The population were 18-65 years old; 3.The population were from residents, primary health care and other common groups; 4.There were clear diagnostic criteria for subthreshold anxiety disorder, and the diagnostic criteria were mainly referred to the diagnostic of anxiety disorder in the Diagnostic and Statistical Manual of Mental Disorders(DSM) or International Classification of Diseases or other related anxiety disorders diagnostic criteria; 5.Reporting the incidence rate or related data of subthreshold anxiety disorder; 6.If the study population of different literatures is the same group and the diagnostic criteria of subthreshold anxiety disorder are not consistent, they will be included, otherwise only the study with the longest follow-up time will be included. Types of study to be excluded:1.Meta, conference papers; 2.The diagnose the subthreshold anxiety disorder only by scale; 3.The primary diagnosis was non subthreshold anxiety disorder or the population comorbid somatic disease.

Intervention: No.

Comparator: No.

Study designs to be included: Corss study or Cohort study.

Eligibility criteria: Types of study to be included: 1.The language of the article is English; 2.The population were 18-65 years old; 3.The population were from residents, primary health care and other common groups; 4.There were clear diagnostic criteria for subthreshold anxiety disorder, and the diagnostic criteria were mainly referred to the diagnostic of anxiety disorder in the Diagnostic and Statistical Manual of Mental Disorders(DSM) or International Classification of Diseases or other related anxiety disorders diagnostic criteria; 5.Reporting the incidence rate or related data of subthreshold anxiety disorder; 6.If the study population of different literatures is the same group and the diagnostic criteria of subthreshold anxiety disorder are not consistent, they will be included, otherwise only the study with the longest follow-up time will be included. Types of study to be excluded:1.Meta、 conference papers; 2.The diagnose the subthreshold anxiety disorder only by scale; 3.The primary diagnosis was non subthreshold anxiety disorder or the population comorbid somatic disease.

Information sources: Four databases were searched: Cochrane Library, PubMed, EMBASE and Web of Science from their inception to June 02, 2020.We serach studies in following MeSH-Terms: (Anxiety OR anxiety disorder OR anxiety disorders OR phobia OR specific phobia OR specific phobias OR social phobia OR social anxiety disorder OR social anxiety disorders OR agoraphobia OR panic disorder OR panic disorders OR generalized anxiety disorder OR generalized anxiety disorders OR pediatric anxiety OR pediatric anxiety disorder OR pediatric anxiety disorders OR separation anxiety disorder OR separation anxiety disorders) AND (Subthreshold OR Subclinical OR Subsyndromal OR Subdiagnostic OR Sub-threshold OR Subclinical OR Sub-syndromal OR Subdiagnostic).

Main outcome(s): The prevalence of subthreshold anxiety disorder in adult. And exploreing the stability of subthreshold anxiety disorder by investigation the prevalence of subthreshold anxiety disorder change into threshold anxiety disorder group or health group.

Quality assessment / Risk of bias analysis: We will take the Risk of Bias Tool to do the quality assessment.

Strategy of data synthesis: We will summarized and presented followed data in a table: first author name, country, age, 
sex, assessment tool, sampling method, case of subthreshold anxiety disorder, number of total sample, the types of subthreshold anxiety disorder. The studies that meet the inclusion criteria will be present data by two independent authors. The data will be analyzed by prevalence of subthreshold anxiety disorder with 95\% confidence intervals using R.3.6.3. Pooledeffect estimates using a random-effects model with Mantel Haenszel statistics. Study heterogeneity was determined using the $I^{2}$ statistic. If $I^{2} \geq 50 \%$ and/or $P<0.1$, we will take random effect model to analysis data; otherwise, we will take fixed effect model.

Subgroup analysis: Comparing the prevalence of subthreshold anxiety disorder of different country, assessment tool, sex, sampling method.

Sensibility analysis: If necessary, sensitivity analysis using standards will be performed.

Language: English.

Country(ies) involved: China.

Keywords: prevalence; subthreshold anxiety disorder.

Contributions of each author:

Author 1 - Cong Zeng - Author 1 drafted the manuscript and did searched strategy, selection of studies and analysis of results. Author 2 - Xiaoyan Yang - The author did selection of studies, data extraction and analysis of results.

Author 3 - Jianqin Cao - She controlled the quality of the research and provided feedback and approved the final manuscript. 